\title{
Triarylmethyl Cation Catalysis: A Tunable Lewis Acid Organo- catalyst for the Synthesis of Bisindolylmethanes
}

\author{
Nicholas G. Boekell \\ Dana J. Cerone \\ Maria M. Boucher \\ Phong K. Quach \\ Wilfried B. Nganyak Tentchou \\ Christine G. Reavis \\ Ifeanyi I. Okoh \\ Jordan O. A. Reid \\ Hayley E. Berg \\ Briana A. Chang \\ Cheyenne S. Brindle*
}

Trinity College, 300 Summit Street, Hartford, CT 06106, USA cheyenne.brindle@trincoll.edu
Received: 29.06.2017

Accepted after revision: 07.08.2017

Published online: 22.08 .2017

DOI: 10.1055/s-0036-1588559; Art ID: so-2017-d0028-I

License terms: (c)

Abstract Triarylmethyl cations serve as tunable organocatalysts for the synthesis of bisindolylmethanes. The catalyst structure can be modified to increase or decrease reactivity as needed to match the requirements of the substrate. High yields are achieved for a variety of substrates by using these green catalysts. Catalyst tuning allows for the use of less reactive electrophiles by increasing the reactivity of the catalyst. Acid-sensitive products can be isolated under these mild reaction conditions.

Key words antibiotics, carbocation, catalysis, electrophilic aromatic substitution, green chemistry, indoles, Lewis acids

The development of carbon-based catalysts, or organocatalysts, has flourished in recent years as alternatives to metal-based catalysts, which are frequently costly, sensitive to air and moisture, and toxic. ${ }^{1-4}$ The majority of these green chemistry efforts have focused on the creation of nucleophilic $^{5,6}$ or Brønsted acid catalysts, such as proline ${ }^{7-9}$ and organophosphoric acids. ${ }^{10-12}$ Research focused on the development of organocatalytic Lewis acids has received far less attention, despite reports of the efficacy of triarylmeth$\mathrm{yl}$ or trityl cation catalysis in the 1980 s. ${ }^{13-15}$ Recent reports by Franzén and co-workers have revived interest in organocatalytic Lewis acid catalysis. ${ }^{16}$ Reports of their utility for Diels-Alder reactions, conjugate additions, ${ }^{17} \alpha$-halogenation, epoxide rearrangements, intramolecular ene reactions, ${ }^{18}$ and an unusual oxo-metathesis reaction ${ }^{19}$ have been described. Recently, the use of chiral counterions has allowed for enantioselective catalysis of a Diels-Alder reac-

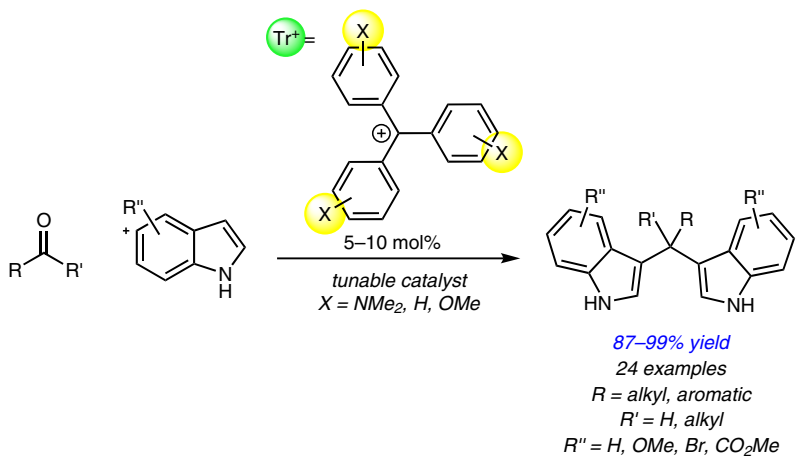

tion. ${ }^{20}$ These successes may portend the applicability of these organocatalysts as general Lewis acid catalysts for a wide range of applications.

One of the main advantages of this class of Lewis acids is the ability to tune their reactivity by changing the identity of the aryl substituents (Figure 1). Indeed, the stability of trityl cations has been shown to span over eight orders of magnitude. ${ }^{21}$ Electron-donating substituents stabilize the cation, reducing its reactivity toward nucleophiles. Conversely, adding electron-withdrawing groups decreases cation stability, increasing reactivity toward nucleophiles.
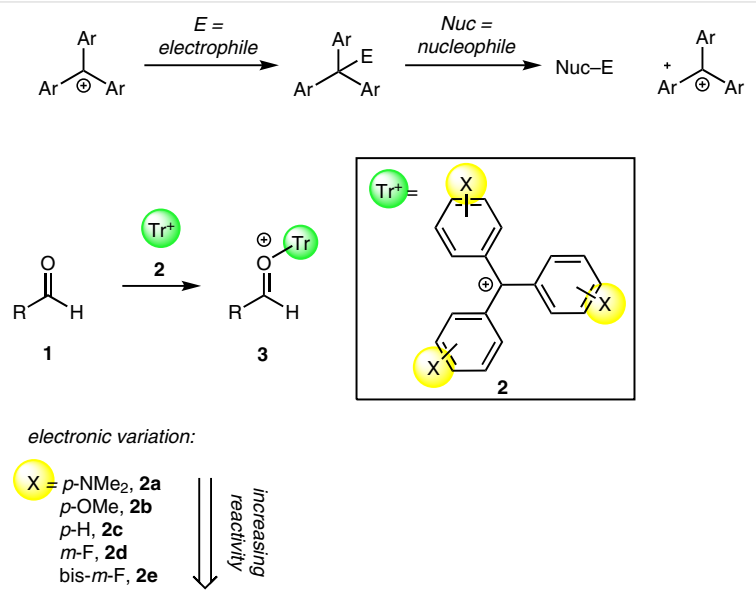

Figure 1 Trityl catalysts are electronically tunable catalysts that can potentially serve as a general Lewis acid activator of electrophiles, including aldehydes

This uniquely wide range of reactivity may allow for catalysis of reactions with very disparate activation barriers using the same molecular scaffold. This remarkable feature could lead to a general Lewis acid catalyst for a wide array 
of nucleophile/electrophile combinations. This is especially useful in gaining specificity in situations when multiple reaction pathways are available. The ability to increase or decrease reactivity is also important to gain generality of reaction conditions across a wide scope of reaction partners. To probe these two features, we chose to look at the Friedel-Crafts reaction of indoles with aldehydes, with a wide array of electronic properties and chemical sensitivities, to investigate whether trityl cations can behave as selective and tunable organocatalysts. The products of these reactions, bisindolylmethanes, have a wide array of biological activities, including antibiotic and antitumor properties. ${ }^{22}$ This important family of compounds has garnered significant attention from synthetic chemists, and various successful methods have been disclosed, ${ }^{23}$ including several green methods. ${ }^{24,25}$ The proposed mechanism of the tritylcatalyzed Friedel-Crafts reaction begins with activation of the aldehyde (1) by the trityl cation (2) to give activated aldehyde $\mathbf{3}$ (Scheme 1 ). Indole (4) addition gives adduct $\mathbf{5}$, which can eliminate trityl alcohol to give the unsaturated adduct $\mathbf{6}$. A second indole (3) attack gives bisindolyl adduct 7. Rearomatization delivers the final bisindolylmethane $\mathbf{8}$ and regenerates the catalyst $\mathbf{2}$ and a molecule of water. The cation must release water to complete this last portion of the catalytic cycle. The ability to tune the catalyst will be essential to balance electrophile activation with stability toward nucleophilic attack by water. Therefore, this reaction is a good probe for the applicability of these tunable organocatalysts in synthesis.

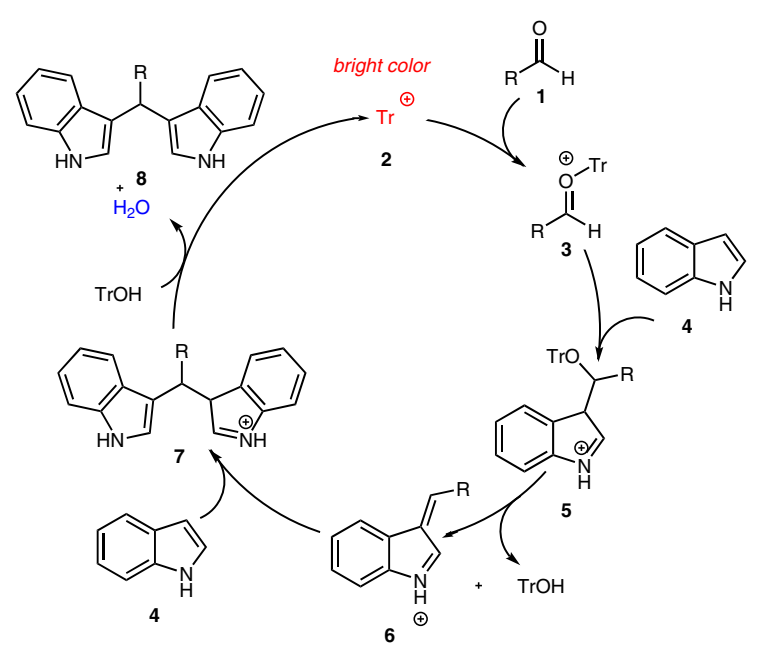

Scheme 1 Proposed reaction mechanism for the trityl (Tr) cation catalyzed bisindolylmethane synthesis

Trityl cations are used commercially as dyes, and therefore the color of the reaction can serve as a visual cue to the state of the catalyst. When the catalyst is quenched by nucleophilic attack at the central carbon atom, conjugation is interrupted, and the molecule no longer absorbs visible light. This property is particularly applicable in the context of bisindolylmethane synthesis, due to the generation of a molecule of water as a by-product in the final step of the catalytic cycle. If regeneration of the catalyst does not occur from the trityl alcohol during the rearomatization step of adduct $\mathbf{7}$, the cation will function as a reagent rather than as a catalyst. This can be seen visually by a fading or complete disappearance of the reaction color. All intermediates in which the central carbon atom of the trityl catalyst is $\mathrm{sp}^{3}$-hybridized are expected to be colorless, but if any free catalyst $\mathbf{2}$ is present, the color should persist. As these dyes can be detected visually, even at very low concentrations, active trityl catalyst $\mathbf{2}$ should be visually apparent. This visual indication of catalyst performance is one of the benefits of using trityl cations as organocatalysts.

To test the effectiveness of trityl catalysts in the synthesis of bisindolylmethanes $\mathbf{8}$, we first investigated the reaction between indole $\mathbf{4}$ and benzaldehyde $\mathbf{1 a}$ (Table 1). With no catalyst, only a trace amount of bisindolylmethane $\mathbf{8 a}$ was present after 2 days (entry 1 ). In contrast, triphenylmethyl tetrafluoroborate $\mathbf{2 c}$, made in situ by reacting triphenylmethanol with fluoroboric acid, gave $71 \%$ yield when used in $5 \mathrm{~mol} \%$ after 5 hours (entry 2). The reaction appeared to slow after an initial burst of activity and the color of the reaction faded. This indicated that the active catalyst was no longer present, so the reactivity of the catalyst was tuned down by using a pre-catalyst with electron-donating substituents on the aromatic rings. Tri-p-tolylmethyl tetrafluoroborate $\mathbf{2 f}$ gave only $45 \%$ yield of bisindolylmethane $\mathbf{8 a}$ (entry 3), but tri-p-methoxyphenylmethyl tetrafluoroborate gave bisindolylmethane 8a in 61\% yield (entry 4). Switching to the even more stabilized tri-p-(dimethylamino)phenylmethyl chloride, commercially sold as crystal violet 2a, gave reduced activity and provided the product 8a in only $38 \%$ yield (entry 5). Decreasing the number of electron-donating groups from three to two, dramatically improved the yield of bisindolylmethane 8a to 95\% (entry 6). This cation is also commercially available as the chloride salt $\mathbf{2 g}$, known as malachite green. Increasing the temperature gave a slightly faster reaction, and the product was obtained in 99\% yield after 5 hours (entry 7). Dichloromethane is not an optimal solvent in terms of creating green reaction methodology, so alternate solvents with less toxicity were explored. Acetonitrile gave $83 \%$ yield at ambient temperature (entry 8 ), but only $65 \%$ yield at $80{ }^{\circ} \mathrm{C}$ (entry 9 ). Ethyl acetate gave $50 \%$ yield at ambient temperature (entry 10 ) and an improved $83 \%$ yield at $75{ }^{\circ} \mathrm{C}$ (entry 11). Ethanol gave $83 \%$ yield at ambient temperature (entry 12 ), but only $64 \%$ yield at $75^{\circ} \mathrm{C}$ (entry 13 ). The performance of dichloromethane was not matched by the greener solvents employed, but both acetonitrile and ethanol at ambient temperature gave useful results, should solvent toxicity be a primary concern for the user. 
Table 1 Tuning the Reaction Conditions for Bisindolylmethane Synthesis using Benzaldehyde and Indole ${ }^{a}$
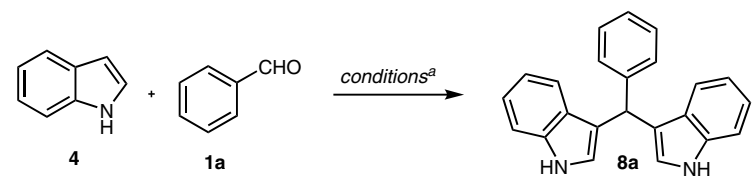

\begin{tabular}{llllll}
\hline Entry & Catalyst & & Solvent & Temp. $\left({ }^{\circ} \mathrm{C}\right)$ & Yield $(\%)^{\mathrm{b}}$ \\
\hline $1 \mathrm{c}$ & none & & $\mathrm{CH}_{2} \mathrm{Cl}_{2}$ & $\mathrm{RT}$ & trace \\
2 & $\mathrm{Ph}_{3} \mathrm{CBF}_{4}$ & $\mathbf{2 c}$ & $\mathrm{CH}_{2} \mathrm{Cl}_{2}$ & $\mathrm{RT}$ & 71 \\
3 & $\left(\mathrm{pMeC}_{6} \mathrm{H}_{5}\right)_{3} \mathrm{COH}, \mathrm{HBF}_{4} \cdot \mathrm{OEt}_{2}$ & $\mathbf{2 f}$ & $\mathrm{CH}_{2} \mathrm{Cl}_{2}$ & $\mathrm{RT}$ & 45 \\
4 & $\left(\mathrm{PMeC}_{6} \mathrm{H}_{5}\right)_{3} \mathrm{COH}, \mathrm{HBF}_{4} \cdot \mathrm{OEt}_{2}$ & $\mathbf{2 b}$ & $\mathrm{CH}_{2} \mathrm{Cl}_{2}$ & $\mathrm{RT}$ & 61 \\
5 & $\left(p \mathrm{NMe}_{2} \mathrm{C}_{6} \mathrm{H}_{5}\right)_{3} \mathrm{CCl}$ & $\mathbf{2 a}$ & $\mathrm{CH}_{2} \mathrm{Cl}_{2}$ & $\mathrm{RT}$ & 38 \\
6 & $\left(p \mathrm{NMe}_{2} \mathrm{C}_{6} \mathrm{H}_{5}\right)_{2} \mathrm{PhCCl}$ & $\mathbf{2 g}$ & $\mathrm{CH}_{2} \mathrm{Cl}_{2}$ & $\mathrm{RT}$ & 95 \\
7 & $\left(p \mathrm{NMe}_{2} \mathrm{C}_{6} \mathrm{H}_{5}\right)_{2} \mathrm{PhCCl}$ & $\mathbf{2 g}$ & $\mathrm{CH}_{2} \mathrm{Cl}_{2}$ & 35 & 99 \\
8 & $\left(p \mathrm{NMe}_{2} \mathrm{C}_{6} \mathrm{H}_{5}\right)_{2} \mathrm{PhCCl}$ & $\mathbf{2 g}$ & $\mathrm{MeCN}_{2}$ & $\mathrm{RT}$ & 83 \\
9 & $\left(p \mathrm{NMe}_{2} \mathrm{C}_{6} \mathrm{H}_{5}\right)_{2} \mathrm{PhCCl}$ & $\mathbf{2 g}$ & $\mathrm{MeCN}_{2}$ & 80 & 65 \\
10 & $\left(p \mathrm{NMe}_{2} \mathrm{C}_{6} \mathrm{H}_{5}\right)_{2} \mathrm{PhCCl}$ & $\mathbf{2 g}$ & $\mathrm{EA}$ & $\mathrm{RT}$ & 50 \\
11 & $\left(p \mathrm{NMe}_{2} \mathrm{C}_{6} \mathrm{H}_{5}\right)_{2} \mathrm{PhCCl}$ & $\mathbf{2 g}$ & $\mathrm{EA}$ & 75 & 83 \\
12 & $\left(p N M{ }_{2} \mathrm{C}_{6} \mathrm{H}_{5}\right)_{2} \mathrm{PhCCl}$ & $\mathbf{2 g}$ & $\mathrm{EtOH}$ & $\mathrm{RT}$ & 83 \\
13 & $\left(p N M{ }_{2} \mathrm{C}_{6} \mathrm{H}_{5}\right)_{2} \mathrm{PhCCl}$ & $\mathbf{2 g}$ & $\mathrm{EtOH}$ & 75 & 64 \\
\hline
\end{tabular}

a Reaction conditions: indole 4 ( 0.2 M, 2 equiv), benzaldehyde 1a (1.1 equiv), catalyst ( $5 \mathrm{~mol} \%), 5 \mathrm{~h}$.

b Isolated yield.

c 2 days.

We next explored the substrate scope of the FriedelCrafts reaction using the optimized conditions (Scheme 2). Electron-poor aromatic aldehydes are good substrates for the malachite green catalysis conditions, yielding the $p$ chloro substituted adduct $\mathbf{8 b}$ in $95 \%$ yield after 2 hours. Increasing the electron-withdrawing capacity further gave even better results, with the $p$-cyano $\mathbf{8 c}$ and $p$-trifluoromethyl $\mathbf{8 d}$ adducts obtained in $99.9 \%$ and $91 \%$ yield, respectively, after only 30 minutes. 2-Naphthaldehyde was also a good substrate, yielding $94 \%$ of adduct 8 e after 1 hour. Electron-rich substrates were expected to give lower activity, and this was indeed the case. The $p$-methoxy adduct $8 \mathbf{f}$ was obtained in $96 \%$ yield after 3 hours, whereas the $p$ thiomethyl $8 \mathrm{~g}$ adduct was isolated in $90 \%$ yield after 5 hours. Variation of the substitution pattern was investigated using $0-, m$-, and $p$-tolylaldehyde. Adducts $\mathbf{8 h}-\mathbf{j}$ were all isolated in high yield, but longer reaction times were necessary for the $o$-substitution pattern, presumably due to steric hindrance. This was also seen for the 2-trifluoromethyl adduct $\mathbf{8 k}$, which was isolated in $95 \%$ yield after 1 day. Competitive binding of the catalyst by the pyridine nitrogen is possible during the synthesis of adduct 81, which is likely the reason for the more sluggish reaction observed with the substrate. Despite the longer reaction time, useful yields can still be obtained despite the competitive binding that is expected to occur with the basic nitrogen of the 2-pyridinecarboxyaldehyde and the product adduct 81 . The reaction methodology is also applicable for free $\mathrm{OH}$ and $\mathrm{NH}$ bonds, yielding adducts $\mathbf{8 m}$ and $\mathbf{8 n}$ in $91 \%$ and $96 \%$ yield, respectively. Aliphatic aldehydes are also tolerated, with isovaleraldehyde yielding adduct 80 in $90 \%$ yield after 2 hours. Increasing the steric hindrance greatly reduced activity. Valeraldehyde adduct $\mathbf{8 p}$ was obtained in only $52 \%$ yield after 1 day. As expected from the results of sterically hindered aldehydes, ketones were found to be very slow to react. Cyclohexanone derivative $\mathbf{8 q}$ was obtained in only $61 \%$ yield after 4 days.

Variation of the indole component was well-tolerated. 5 -Bromoindole yielded adduct $8 \mathbf{r}$ in $87 \%$ yield after $45 \mathrm{~min}$ utes. Adding electron-donating groups gave a very fast reaction, with 5-methoxyindole adduct 8s produced in 95\% yield after only 15 minutes. An electron-withdrawing ester substituent was well-tolerated, giving $87 \%$ yield of adduct 8t after 1 hour. N-Alkylated adducts $8 \mathbf{u}, 8 \mathbf{v}$, and $8 \mathbf{w}$ were obtained in $95 \%, 90 \%$, and $86 \%$ yield, respectively. Interestingly, 2-methylindole was also a good substrate, yielding adduct $\mathbf{8 x}$ in $99 \%$ yield after only 30 minutes, despite steric hindrance near the reaction center. Adduct $\mathbf{8 x}$ was found to be unstable to silica gel, presumably due to the slight acidity of the chromatographic material. The product was isolated by using our recently developed bisulfite extraction protocol ${ }^{27}$ to purify the product away from the slight excess of aldehyde used in the reaction. After extraction, the product was pure by ${ }^{1} \mathrm{H}$ NMR analysis, thereby avoiding the need for purification using silica gel chromatography. This result highlights both the mildness of the malachite green reaction conditions, and the mildness of the bisulfite work-up protocol, allowing for the application of these methods to sensitive substrates.

To improve the reactivity of poorly reactive substrates in the trityl-catalyzed Friedel-Crafts reaction, we increased the reactivity of the trityl cation employed (Scheme 3). Although malachite green is preferable as a catalyst due to its commercial availability and low cost, for certain substrates enhanced activation was required for the reaction to proceed at a satisfactory rate. Switching to the less electrondonating tri-p-methoxyphenylmethyl cation $\mathbf{2 b}$ increased the yield of $o$-pyridine adduct $\mathbf{8 1}$ to $95 \%$. This triaryl alcohol is commercially available, but can also be readily synthesized in a single step from inexpensive commercial starting materials. ${ }^{29}$ Sterically hindered isobutyraldehyde also participated effectively, giving adduct $\mathbf{8 p}$ in $99 \%$ yield. The reaction was also improved for ketone-derived adduct $\mathbf{8 q}$, which was produced in $98 \%$ yield. 


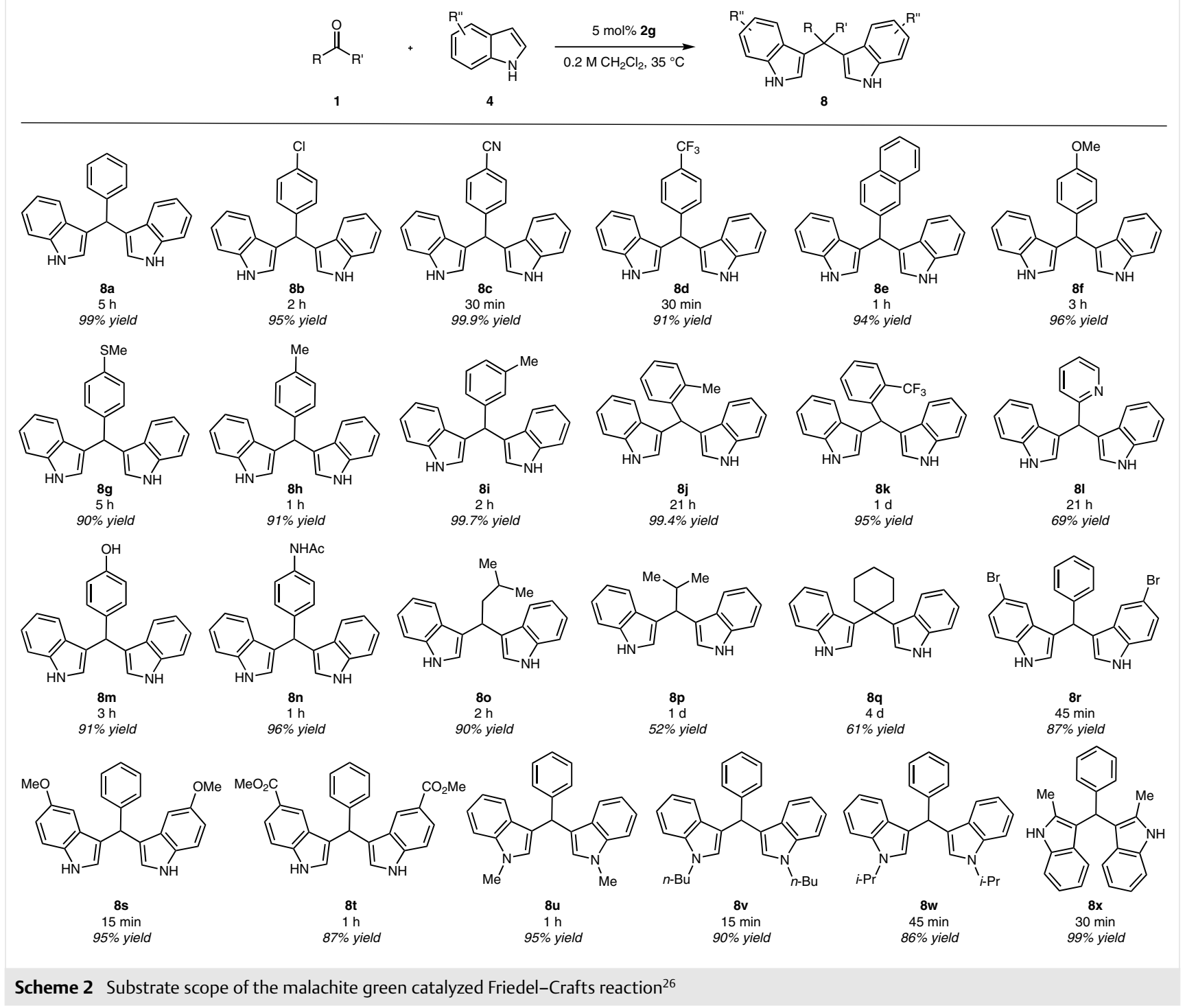

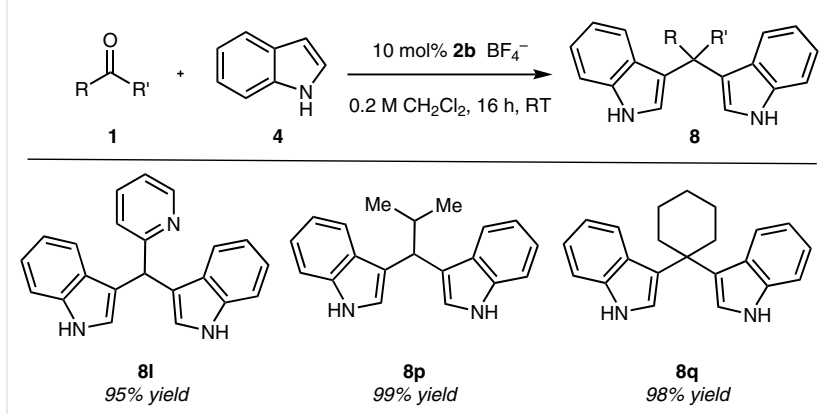

Scheme 3 Catalyst tuning for less electrophilic substrates ${ }^{28}$

Trityl catalysis proves to be an effective and green solution to the challenge of bisindolylmethane synthesis. The tunability of these catalysts allowed for optimization with respect to catalyst stability toward the water generated as a by-product during the catalytic cycle. Malachite green is a convenient, commercially available organocatalyst that is applicable for most substrates tested, but the catalyst can be further optimized for less reactive substrates to enhance reaction rates. These results demonstrate the benefits of tunable Lewis acidic organocatalysts in synthesis.

\section{Funding Information}

Acknowledgment is made to the Donors of the American Chemical Society Petroleum Research Fund for partial support of this research. We are grateful to the National Science Foundation (CHE-0619275 and CHE-0963165) for renovation and instrumentation grants that supported this research. 


\section{Supporting Information}

Supporting information for this article is available online at https://doi.org/10.1055/s-0036-1588559.

\section{References and Notes}

(1) Buckley, B. R. Annu. Rep. Prog. Chem., Sect. B: Org. Chem. 2013, 109, 189.

(2) Bertelsen, S.; Jorgensen, K. A. Chem. Soc. Rev. 2009, 38, 2178.

(3) Liu, D.; Chen, E. Y.-X. Green Chem. 2014, 16, 964.

(4) Shaikh, I. R. J. Catal. 2014, 1.

(5) Enders, D.; Niemeier, O.; Henseler, A. Chem. Rev. 2007, 107, 5606.

(6) Flanigan, D. M.; Romanov-Michailidis, F.; White, N. A.; Rovis, T. Chem. Rev. 2015, 115, 9307.

(7) Liu, J.; Wang, L. Synthesis 2017, 49, 960.

(8) Khandelwal, S.; Tailor, Y. K.; Kumar, M. Curr. Organocatal. 2016, $3,176$.

(9) Mandalapu, D. Synlett 2015, 26, 707.

(10) Monaco, M. R.; Pupo, G.; List, B. Synlett 2016, 27, 1027.

(11) Zhao, R.; Shi, L. ChemCatChem 2014, 6, 3309.

(12) Akiyama, T.; Mori, K. Chem. Rev. 2015, 115, 9277.

(13) Kobayashi, S.; Mukaiyama, T. Chem. Lett. 1986, 15, 221.

(14) Hayashi, M.; Mukaiyama, T. Chem. Lett. 1987, 16, 289.

(15) Denmark, S. E.; Chen, C.-T. Tetrahedron Lett. 1994, 35, 4327.

(16) Naidu, V. R.; Ni, S.; Franzén, J. ChemCatChem 2015, 7, 1896.

(17) Bah, J.; Franzén, J. Chem. Eur. J. 2014, 20, 1066.

(18) Bah, J.; Naidu, V. R.; Teske, J.; Franzén, J. Adv. Synth. Catal. 2015, $357,148$.

(19) Veluru, R. N.; Bah, J.; Franzén, J. Eur. J. Org. Chem. 2015, 1834.

(20) Ni, S.; Ramesh Naidu, V.; Franzén, J. Eur. J. Org. Chem. 2016, 1708.

(21) Horn, M.; Mayr, H. J. Phys. Org. Chem. 2012, 25, 979.

(22) Imran, S.; Taha, M.; Ismail, N. H.; Khan, K. M.; Naz, F.; Hussain, M.; Tauseef, S. Molecules 2014, 19, 11722.

(23) Praveen, P. J.; Parameswaran, P. S.; Majik, M. S. Synthesis 2015, 47, 1827.

(24) Xia, M.; Wang, S.; Yuan, W. Synth. Commun. 2004, 34, 3175.

(25) Xie, Z.-B.; Sun, D.-Z.; Jiang, G.-F.; Le, Z.-G. Molecules 2014, 19, 19665.

(26) General procedure for malachite green-catalyzed reactions: Indole $(23.4 \mathrm{mg}, 0.20 \mathrm{mmol})$, and malachite green $(1.8 \mathrm{mg}$, $0.005 \mathrm{mmol}$ ) were added to a 1 dram vial equipped with a stir bar. Dichloromethane $(0.5 \mathrm{~mL}, 0.2 \mathrm{M})$ was added, followed by the aldehyde $(0.11 \mathrm{mmol}$; liquid aldehydes were directly injected, solid aldehydes were added as a solution in $\mathrm{CH}_{2} \mathrm{Cl}_{2}$ ) and the reaction was then heated to $35{ }^{\circ} \mathrm{C}$. The reaction was stirred and the progress of the reaction was followed by TLC. The crude reaction was then purified by chromatography to isolate the product.

3,3'-(Phenylmethylene)bis(1H-indole) (8a): The reaction time was 5 hours and a $15-25 \%$ ethyl acetate/hexanes gradient was used to purify the crude reaction mixture to give the title compound as a red foam $(29.5 \mathrm{mg}, 99 \%$ yield).

3,3'-((4-Chlorophenyl)methylene)bis(1H-indole) (8b): The reaction time was 2 hours and a 15-35\% ethyl acetate/hexanes gradient was used to purify the crude reaction mixture to give the title compound as a red foam (33.8 $\mathrm{mg}, 95 \%$ yield).

4-(Di(1H-indol-3-yl)methyl)benzonitrile (8c): The reaction time was 30 minutes and a 15-45\% ethyl acetate/hexanes gradient was used to purify the crude reaction mixture to give the title compound as a pink foam (34.5 mg, 99.9\% yield).

3,3'-((4-(Trifluoromethyl)phenyl)methylene)bis(1H-indole)

(8d): The reaction time was 30 minutes and a $15-25 \%$ ethyl acetate/hexanes gradient was used to purify the crude reaction mixture to give the title compound as a red foam (34.6 mg, 91\% yield).

3,3'-(Naphthalen-2-ylmethylene)bis(1H-indole) (8e): The reaction time was 1 hour and a $2-25 \%$ ethyl acetate/hexanes gradient was used to purify the crude reaction mixture to give the title compound as a red foam ( $34.7 \mathrm{mg}, 94 \%$ yield).

3,3'-((4-Methoxyphenyl)methylene)bis(1H-indole) (8f): The reaction time was 3 hours and a $15-40 \%$ ethyl acetate/hexanes gradient was used to purify the crude reaction mixture to give the title compound as an orange foam (33.7 $\mathrm{mg}$, 96\% yield).

3,3'-((4-(Methylthio)phenyl)methylene)bis(1H-indole) (8g): The reaction time was 5 hours and a $10-35 \%$ ethyl acetate/hexanes gradient was used to purify the crude reaction mixture to give the title compound as a red oil ( $32.5 \mathrm{mg}, 90 \%$ yield).

3,3'-(p-Tolylmethylene)bis(1H-indole) $(\mathbf{8 h})$ : The reaction time was 1 hour and a $15-25 \%$ ethyl acetate/hexanes gradient was used to purify the crude reaction mixture to give the title compound as a red foam (30.3 $\mathrm{mg}, 91 \%$ yield).

3,3'-( $\boldsymbol{m}$-Tolylmethylene)bis( $\mathbf{1 H}$-indole) (8i): The reaction time was 2 hours and a 15-35\% ethyl acetate/hexanes gradient was used to purify the crude reaction mixture to give the title compound as a peach foam ( $33.5 \mathrm{mg}, 99.7 \%$ yield).

3,3'-(o-Tolylmethylene)bis $(\mathbf{1 H}$-indole) $(\mathbf{8 j})$ : The reaction time was 21 hours and a $15-30 \%$ ethyl acetate/hexanes gradient was used to purify the crude reaction mixture to give the title compound as a white foam (34.1 $\mathrm{mg}, 99.4 \%$ yield).

3,3'-((2-(Trifluoromethyl)phenyl)methylene)bis(1H-indole)

(8k): The reaction time was 24 hours and a $20-40 \%$ ethyl acetate/hexanes gradient was used to purify the crude reaction mixture to give the title compound as a white foam $(36.7 \mathrm{mg}$, $95 \%$ yield).

4-(Di(1H-indol-3-yl)methyl)phenol $(\mathbf{8 m})$ : The reaction time was 3 hours and a $25-80 \%$ ethyl acetate/hexanes gradient was used to purify the crude reaction mixture to give the title compound as an orange foam (30.2 $\mathrm{mg}, 91 \%$ yield).

$\mathbf{N}$-(4-(Di(1H-indol-3-yl)methyl)phenyl)acetamide (8n): The reaction time was 1 hour and a $20-100 \%$ ethyl acetate/hexanes gradient was used to purify the crude reaction mixture to give the title compound as a red-orange foam ( $36.6 \mathrm{mg}, 96 \%$ yield).

3,3'-(3-Methylbutane-1,1-diyl)bis(1H-indole) (80): The reaction time was 2 hours and a 15-35\% ethyl acetate/hexanes gradient was used to purify the crude reaction mixture to give the title compound as a light-brown foam ( $27.2 \mathrm{mg}$, $90 \%$ yield).

3,3'-(Phenylmethylene)bis(5-bromo-1H-indole) (8r): The reaction time was 45 minutes and a 5-45\% ethyl acetate/hexanes gradient was used to purify the crude reaction mixture to give the title compound as a red-orange foam $(82.0 \mathrm{mg}, 87 \%$ yield).

3,3'-(Phenylmethylene)bis(5-methoxy-1H-indole) (8s): The reaction time was 15 minutes and a 5-30\% ethyl acetate/hexanes gradient was used to purify the crude reaction mixture to give the title compound as a white solid (70.1 mg, 93\% yield).

Dimethyl 3,3'-(Phenylmethylene)bis(1H-indole-5-carboxylate) (8t): The reaction time was 1 hour and a $18-45 \%$ ethyl acetate/hexanes gradient was used to purify the crude reaction mixture to give the title compound as a light-pink solid (74.0 $\mathrm{mg}, 87 \%$ yield).

3,3'-(Phenylmethylene)bis(1-methyl-1H-indole) (8u): This reaction was done on a $0.4 \mathrm{mmol}$ scale. The reaction time was 1 
hour and a $2-10 \%$ ethyl acetate/hexanes gradient was used to purify the crude reaction mixture to give the title compound as a pink foam (133.6 $\mathrm{mg}$, 95\% yield).

3,3'-(Phenylmethylene)bis(1-butyl-1H-indole) (8v): The reaction time was 15 minutes and a 5-20\% ethyl acetate/hexanes gradient was used to purify the crude reaction mixture to give the title compound as a red foam (38.6 mg, 90\% yield).

3,3'-(Phenylmethylene)bis(1-isopropyl-1H-indole) (8w): The reaction time was 45 minutes and a $2-12 \%$ ethyl acetate/hexanes gradient was used to purify the crude reaction mixture to give the title compound as a red film ( $69.0 \mathrm{mg}, 86 \%$ yield).

3,3'-(Phenylmethylene)bis(2-methyl-1H-indole) (8x): The reaction time was 30 minutes and the mixture was then diluted with methanol and washed with sodium bisulfite to remove excess benzaldehyde. ${ }^{26}$ Ethyl acetate: hexanes (1:1) was then added to extract the organic layer, which was then dried $\left(\mathrm{MgSO}_{4}\right)$, filtered, and concentrated in vacuo to give the title compound as a pink foam (35.0 mg, 99\% yield)

(27) Boucher, M. M.; Furigay, M. H.; Quach, P. K.; Brindle, C. S. Org. Process Res. Dev. 2017, DOI: 10.1021/acs.oprd.7b00231.

(28) General Procedure for Reactions using Tri-p-methoxyphenylmethanol as Precatalyst: Tri-p-methoxyphenylmethanol $(0.011 \mathrm{mmol})$ was added to a 1 dram vial equipped with a stir bar and a cap fitted with a septum. Dichloromethane $(0.2$ $\mathrm{mL}$ ) was added, followed by tetrafluoroboric acid diethyl etherate complex $(1.4 \mu \mathrm{L}, 0.010 \mathrm{mmol})$ to give a bright red-orange color. Aldehyde $(0.11 \mathrm{mmol})$ was then added by using a syringe, followed by indole $(23.4 \mathrm{mg}, 0.20 \mathrm{mmol})$ as a solution in dichloromethane $(0.2 \mathrm{~mL}$, and a $0.1 \mathrm{~mL}$ rinse). The reaction was stirred and the progress of the reaction was followed by TLC. The crude reaction was then purified by chromatography to isolate the product.

3,3'-(2-Pyridinylmethylene)bis-(1H-indole) (81): The reaction time was 16 hours and a $40-70 \%$ ethyl acetate/hexanes gradient was used to purify the crude reaction mixture to give the title compound as a white amorphous solid (30.5 mg, 95\% yield).

3,3'-(2-Methylpropylidene)bis(1H-indole) $(\mathbf{8 p})$ : The reaction time was 16 hours and a $10-25 \%$ ethyl acetate/hexanes gradient was used to purify the crude reaction mixture to give the title compound as a white foam ( $28.9 \mathrm{mg}, 99 \%$ yield).

3,3'-(Cyclohexane-1,1-diyl)bis(1H-indole) (8q): The reaction time was 16 hours and a $10-25 \%$ ethyl acetate/hexanes gradient was used to purify the crude reaction mixture to give the title compound as a white foam ( $30.8 \mathrm{mg}, 98 \%$ yield)

(29) Guieu, S.; Rocha, J.; Silva, A. M. S. Tetrahedron Lett. 2013, 54, 2870. 